\title{
RETRACTED ARTICLE: A new deep representation for large-scale scene classification
}

\author{
Bo Dai ${ }^{1} \cdot$ Feng $\mathrm{Mei}^{1} \cdot$ Deliang $\mathrm{Ji}^{2} \cdot$ Caiyou $\mathrm{Zhang}^{1} \cdot$ \\ Jia $\mathrm{Shi}^{2}$
}

Received: 9 October 2017 /Revised: 12 October 2017 / Accepted: 19 October 2017 /

Published online: 28 November 2017

(C) Springer Science+Business Media, LLC 2017

The Editor-in-Chief has retracted this article [1], which was published as part of special issue "Multi-source Weak Data Management using Big Data", because the article shows substantial overlap most notably with the article cited [2]. In addition, there is evidence of figure duplication without appropriate permission, as well as evidence suggesting authorship manipulation and an attempt to subvert the peer review process.

The authors have not responded to correspondence about this retraction.

\section{References}

1. Dai, B., Mei, F., Ji, D. et al. A new deep representation for large-scale scene classification. Multimed Tools Appl (2017). https://doi.org/10.1007/s11042-017-5322-5

2. Zhang, L., Bian, W., Song, M. et al. Integrating local features into discriminative graphlets for scene classification. Lecture Notes in Computer Science (2011). https://doi. org/10.1007/978-3-642-24965-5_74

Electronic supplementary material The online version of this article (https://doi.org/10.1007/s11042-0175322-5) contains supplementary material, which is available to authorized users.

Caiyou Zhang

zhangcaiyou1778@126.com

1 State Grid Zhejiang Electric Power Company Information \& Telecommunication Branch, Hangzhou, China

2 Zhejiang Huayun, Zhejiang, China 